\title{
FASN expression, angiogenesis lymphangiogenesis in central and peripheral giant cell lesions
}

\author{
Saulo Gabriel Moreira FALCl'1 , Ana Terezinha Marques MESQUITA', Bruno Augusto Benevenuto de ANDRADE², \\ João Luiz de MIRANDA ${ }^{1}$, Jorge Esquiche LEÓN³, Oslei Paes de ALMEIDA4, Cássio Roberto Rocha dos SANTOS ${ }^{1}$

\footnotetext{
1- Department of Dentistry, College of Basic Sciences and Health, Federal University of Vales do Jequitinhonha e Mucuri (UFVJM), Diamantina, MG, Brazil. 2- Oral Pathology, School of Dentistry, Fluminense Federal University, Nova Friburgo, RJ, Brazil.

3- Oral Pathology, Department of Stomatology, Public Oral Health and Forensic Dentistry, University of São Paulo, Ribeirão Preto, SP, Brazil.

4- Oral Pathology Section, Department of Oral Diagnosis, Piracicaba Dental School, University of Campinas (UNICAMP), Piracicaba, SP, Brazil.
}

Corresponding Address: Saulo Gabriel Moreira Falci - Tiradentes, 195 - Diamantina - MG - Brazil - Postal code: 39100/000 - Phone/Fax: +55 383532 - 6000 I +55388817 1454 - e-mail: saulofalci@hotmail.com

Submitted: September 5, 2013 - Modification: December 4, 2013 - Accepted: January 20, 2014

\section{ABSTRACT}

\begin{abstract}
Central giant cell lesion (CGCL) and peripheral giant cell lesion (PGCL) are non-neoplastic proliferative processes of the jaws. PGCL is a reactive process induced by irritant local factors and CGCL is an intra-osseous lesion of unknown etiology. Both lesions exhibit similar histologic features showing abundant mononuclear cells, admixed with a large number of multinucleated giant cells and a rich vascularized stroma with extravasated erythrocytes, hemosiderin deposition, and blood-filled pools. Recent studies have linked fatty acid synthase (FASN) with angiogenesis. Objective: To evaluate angiogenesis and lymphangiogenesis and their relationship with FASN expression in CGCL and PGCL. Material and Methods: Thirteen CGCL and 14 PGCL of the jaws were selected for immunoexpression of FASN; CD34 and CD105 (to assess blood microvessel density [MVD] and microvessel area [MVA]); and D2-40 (to assess lymphatic MVD and MVA). Results: Within PGCL and CGCL, MVD-CD34 was significantly higher than MVD-CD105, followed by MVD-D2-40. Moreover, a significantly higher number of FASN-positive multinucleated giant cells than mononuclear cells were observed. Between PGCL and CGCL, only MVD-CD34 and all MVA were significantly higher in PGCL. Positive correlation between MVA-CD105 with FASNpositive mononuclear cells in both lesions was observed. Conclusions: Our results show both lesions exhibiting similar levels of FASN expression and neoangiogenesis, suggesting constitutive processes that regulate tissue maintenance.
\end{abstract}

Keywords: Giant cell lesion. Immunohistochemistry. Angiogenesis. Lymphangiogenesis. Fatty acid synthase.

\section{INTRODUCTION}

Giant cell lesion (GCL) of the jaws is a nonneoplastic proliferative process, divided into central giant cell lesion (CGCL) and peripheral giant cell lesion (PGCL). PGCL is considered a reactive process induced by local irritants on the gingiva or alveolar mucosa. CGCL is an intra-osseous lesion of unknown etiology ${ }^{11}$. Both CGCL and PGCL exhibit similar histopathological features, and are characterized by the presence of abundant mononuclear stromal cells, admixed with a large number of multinucleated giant cells and a rich vascularized stroma with extravasated erythrocytes, hemosiderin deposition, and bloodfilled pools. In spite of this, these lesions may have different clinical behaviors ${ }^{11,13,23}$.

Fatty acid synthase (FASN) is the metabolic enzyme responsible for endogenous synthesis of saturated long-chain fatty acid, specifically palmitate, from the precursors acetyl-CoA and malonyl-CoA ${ }^{7}$. FASN is overexpressed in a variety of human cancers affecting breast ${ }^{19}$, ovaries ${ }^{2}$, prostate $^{20}$, and oral cavity ${ }^{22}$, whereas FASN is 
downregulated in most normal human tissues (except in the liver, lactating breast, fetal lung, and adipose tissue) because cells preferentially use circulating dietary fatty acids for the synthesis of new structural lipids ${ }^{29}$. In the oral cavity, FASN expression has been shown in squamous cell carcinoma and melanoma3,22; however, its expression in benign neoplasms ${ }^{3}$ and/or reactive conditions is little known. Interestingly, some studies have linked FASN expression with endothelial cell proliferation ${ }^{4,21}$. Accordingly, the role of FASN expression in angiogenesis must be better defined. To our knowledge, FASN reactivity in GCL of the jaws is unknown.

CD34 is a cell surface glycoprotein consistently expressed in the vascular endothelium. Some studies have previously assessed CD34 expression in CGCL in order to compare aggressive and non-aggressive subtypes. They showed increased microvessel density (MVD)-CD34 in aggressive $\mathrm{CGCL}^{10,18,25}$. Although CD34 is unable to distinguish between pre-existing vessels and neoformed vessels, interestingly, it has been shown that distinguishing neoformed vessels in proliferative tissues is relevant and may have prognostic implications, identifying possible targets for developing anti-angiogenic therapeutic strategies ${ }^{5,18}$. CD105 (endoglin) is an angiogenic membrane protein that is highly expressed in neoformed vessels ${ }^{6}$. Although CD105 has been assessed in oral vascular malformations and pyogenic granulomas ${ }^{26}$, to our knowledge, CD105 expression in GCL of the jaws is unknown. Another unclear and relevant point is the characterization of the lymphatic MVD (LMVD) in GCL, which may be immunohistochemically evaluated through the D240 marker $^{13}$. Nevertheless, as in the case of CD105, the lymphatic vascular stroma characterization in GCL of the jaws has not been evaluated.

As previously mentioned, increased FASN expression in oral malignant tumors has been reported ${ }^{3,22}$, and some studies have linked FASN expression with endothelial cell proliferation ${ }^{4,21}$. It remains to be determined what happens with such events in oral benign and/or reactive lesions. Thus, the aim of the current study was to assess angiogenesis and lymphangiogenesis, as well as establishing their relationship with FASN expression in CGCL and PGCL of the jaws.

\section{MATERIAL AND METHODS}

This retrospective study examined the records and tissues of patients diagnosed and treated for GCL of the jaws. None of the patients had received any treatment with a therapeutic agent prior to the time of the biopsy procedure. Formalin-fixed, paraffin-embedded tissue blocks of $13 \mathrm{CGCL}$ ( 8 males, 5 females; mean age 18.5 years; 8 mandible, 5 maxilla) and 14 PGCL (9 males, 5 females; mean age 38.9 years; 11 mandibular gingiva, 3 maxillary gingiva) were selected from our laboratory archives. According to their clinical characteristics, such as painful symptoms, growth pattern rate, root resorption, cortical bone perforation and recurrence, the CGCL cases of the current study were classified as non-aggressive ${ }^{8}$. All lesions were reviewed through hematoxylineosin stained slides and the diagnosis was confirmed independently by two authors. Excluded from the study were GCL cases which presented inadequate clinical description, cases diagnosed as aneurysmal bone cyst, cherubism and brown tumor of hyperparathyroidism (confirmed by establishing elevated serum parathyroid hormone levels), as well as those with insufficient material for histological analysis. This study was approved by the Research Ethics Committee (Process 042/2011).

\section{Immunohistochemical methods}

For each antibody (FASN, CD34, CD105 and D2$40), 3-\mu m$-thick sections mounted on silane-coated glass slides were used. All tissue specimens were fixed in $10 \%$ neutral-buffered formalin for 24 hours at room temperature, embedded in paraffin at $55^{\circ} \mathrm{C}$, and cut into parallel consecutive sections. For the immunohistochemical (IHC) reactions, the slides were hydrated and treated with hydrogen peroxide (3\%). Primary antibodies, dilutions and antigen retrieval are shown in Table 1 . The tissue sections were then washed three times in phosphate buffered saline (PBS) solution and exposed to secondary antibody using the LSAB+Kit (Dako, Carpinteria, CA, USA). Peroxidase activity was visualized by using the chromogen diaminobenzidine (DAB)

Table 1- Antibodies used for immunohistochemical analysis of central and peripheral giant cell lesions

\begin{tabular}{ccccc}
\hline Antibody & Clone & Manufacturer & Dilution & Antigen retrieval \\
\hline FASN & $23 / 610962$ & $\begin{array}{c}\text { Becton Dickinson Transduction } \\
\text { Laboratories }^{1}\end{array}$ & $1: 200$ & TRIS/EDTA (pH 9.0) \\
CD34 & QBEnd10 & Dako\# & jan/ 1:50 & Citric acid (pH 6.0) \\
CD105 & SN6h & Dako & jan/ 1:30 & Proteinase K \\
D2-40 & D2-40 & Dako & $1: 100$ & Citric acid (pH 6.0) \\
\hline
\end{tabular}

${ }^{1}$ Lexington, KY, USA. \# Carpinteria, CA, USA 
(Sigma Chemical Co., St. Louis, USA). Finally, the tissue sections were counterstained with Carrazi's hematoxylin for 5 minutes. Thereafter, the sections were dehydrated in a series of graded ethanol solutions, diaphanized and mounted in Canada balsam under cover glasses. Sections of oral Kaposi's sarcomas were included in all reactions as positive control for CD34, CD105 and D2-40, while prostate carcinomas were used for FASN. Negative controls of reactions were performed by omitting the primary antibody. The slides were scanned and photographed using the Aperio Scanscope CS Slide Scanner (Aperio Technologies ${ }^{\circledR}$, Vista, CA, USA).

\section{Immunostaining assessment and statistical analysis}

MVD and microvessel area (MVA), assessed through CD34 and CD105 expression, as well as lymphatic MVD (LMVD) and MVA (LMVA), assessed through D2-40 expression, were established identifying the most vascularized areas within the lesions ("hot spots"), which were chosen at low magnification (100x) and subsequently photographed in five high-power fields (at 400x magnification $)^{14}$. The images were made using the Aperio Scan Scope ${ }^{\circledR}$ software, which depicted the image size (height: $580 \mu \mathrm{m} /$ width: $320 \mu \mathrm{m}$ ), resulting in a total area of $185,600 \mu \mathrm{m}^{2}$.

MVD and MVA were defined as the number and area, respectively, of positive microvessels stained per field. Sequentially, the mean values of the five high-power fields were calculated to assess the final MVD and MVA. The MVA measurement was done by means of vascular contour tracing using the
Image ${ }^{\circledR}$ software, which automatically measured the analyzed area $\left(\mu \mathrm{m}^{2}\right)$. Single endothelial cells or clusters of endothelial cells, with or without a lumen, were considered to be individual vessels.

To assess FASN expression, similar to angiogenic markers, the area containing a high number of immunostained cells was identified and selected at low magnification (100x) in both lesions, and subsequently photographed in five high-power fields (at 400x magnification). The total number of FASNpositive mononuclear cells and multinucleated giant cells in both lesions were counted in each high-power field using the software Image ${ }^{\circledR}{ }^{\circledR}$ (Scion Corporation, USA), and then the average percentages of the immunostained cells were calculated in each case.

The data collected were recorded and organized in a database using Statistical Package for Social Science (version 17.0; SPSS Inc., Chicago, IL, USA). Normal distribution was tested using the ShapiroWilk test. In samples with a normal distribution, the Student t-test and Pearson correlation were applied. In samples that presented non-normal distributions, the Mann-Whitney $U$ test and Spearman correlation were applied. A $\mathrm{P}<0.05$ probability value was considered statistically significant.

\section{RESULTS}

All vascular structures in all cases were highlighted by CD34. By comparing the MVD within PGCL and CGCL, a significantly lesser number of CD105-positive vessels than CD34-positive vessels $(P<0.001)$ was observed (Table 2$)$. In addition, a

Table 2- Comparison between PGCL and CGCL in relation to (L)MVD, (L)MVA, and percentage of FASN-positive multinucleated giant cells and mononuclear cells

\begin{tabular}{|c|c|c|c|c|c|}
\hline Variable & $\mathbf{n}$ & $p$ & Variable & $\mathbf{n}$ & $\mathbf{p}$ \\
\hline PGCL & & & CGCL & & \\
\hline MVD-CD34 & 14 & $<.001$ & MVD-CD34 & 13 & $<.001$ \\
\hline MVD-CD105 & 14 & & MVD-CD105 & 13 & \\
\hline MVD-CD34 & 14 & $<.001$ & MVD-CD34 & 13 & .001 \\
\hline LMVD-D2-40 & 14 & & LMVD-D2-40 & 13 & \\
\hline MVD-CD105 & 14 & .015 & MVD-CD105 & 13 & .008 \\
\hline LMVD-D2-40 & 14 & & LMVD-D2-40 & 13 & \\
\hline FASN+ MGC & 14 & .002 & FASN+ MGC & 13 & .004 \\
\hline $\mathrm{FASN}+\mathrm{MC}$ & 14 & & $\mathrm{FASN}+\mathrm{MC}$ & 13 & \\
\hline MVA-CD34 & 14 & $<.001$ & MVA-CD34 & 13 & $<.001$ \\
\hline MVA-CD105 & 14 & & MVA-CD105 & 13 & \\
\hline MVA-CD34 & 14 & $<.001$ & MVA-CD34 & 13 & $<.001$ \\
\hline LMVA-D2-40 & 14 & & LMVA-D2-40 & 13 & \\
\hline MVA-CD105 & 14 & $<.001$ & MVA-CD105 & 13 & $<.001$ \\
\hline LMVA-D2-40 & 14 & & LMVA-D2-40 & 13 & \\
\hline
\end{tabular}


significant lesser number of D2-40-positive vessels than CD105-positive vessels in PGCL $(P=0.015)$ and $\operatorname{CGCL}(P=0.008)$ was detected. This latter in percentage indicates that $31 \%$ and $13 \%$ of the vessels in PGCLs, and $33 \%$ and $8 \%$ of the vessels in CGCLs were positive for CD105 and D2-40, respectively.

The number of CD34-positive vessels was significantly greater in PGCL than CGCL $(P=0.002)$ (Figures 1a, 1b; Figure 2a). However, no statistical difference regarding the number of CD105- and D240-positive vessels was found when comparing both lesions (Figures 1c, 1d; Figure 2a; Figures 3a, 3b).
In relation to vessel luminal area, a significantly greater MVA for all vascular markers used in PGCL than CGCL was observed (CD34, $p=0.001$; CD105, $p=0.041$ and D2-40, $p=0.036$ ) (Figure 2b). Moreover, when comparing the MVA within PGCL and CGCL, a significantly lesser luminal area of CD105-positive vessels than CD34-positive vessels $(P<0.001)$ was observed. In addition, it was detected a significant lesser luminal area of D2-40-positive vessels than CD105-positive vessels in both lesions $(P<0.001)$ (Table 2$)$.

Moreover, through consecutive section analysis, we found focal areas showing vessels stained with

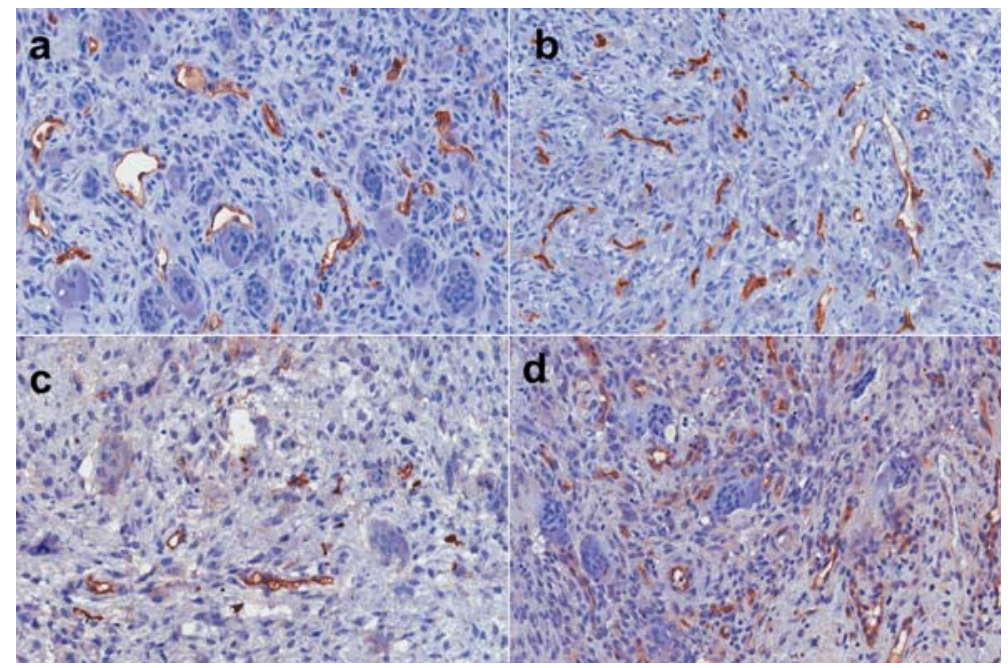

Figure 1- Immunoexpression of CD34 in CGCL (a, 400x) and PGCL (b, 400x), almost all vessels were CD34-positive in both lesions. Immunoexpression of CD105 in CGCL (c, 400x) and PGCL (d, 400x). Although not statistically significant, a lesser number of CD105-positive vessels was observed in CGCL than in PGCL
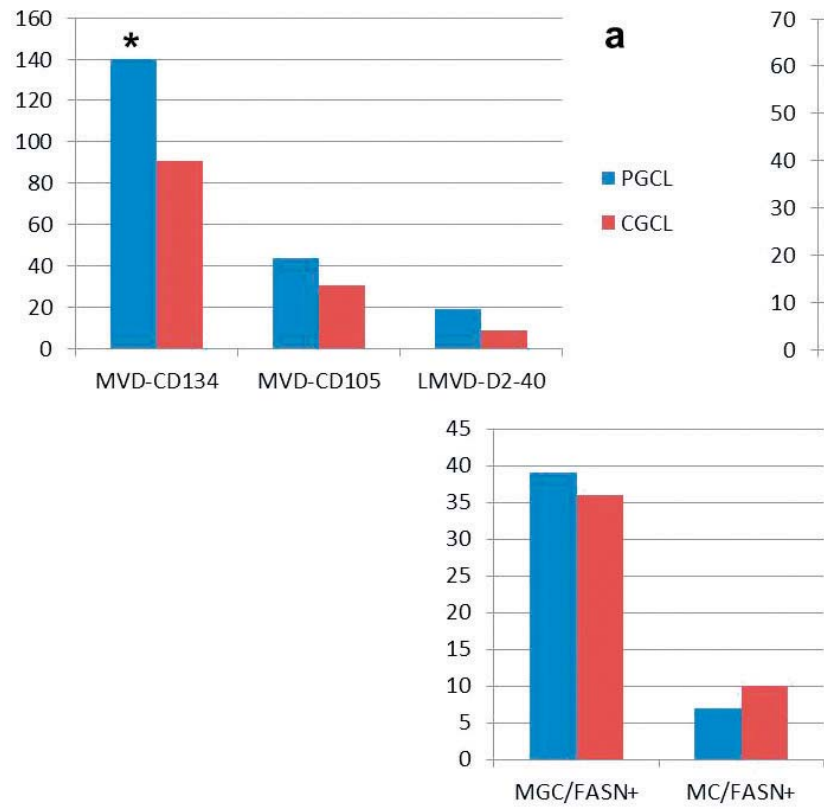

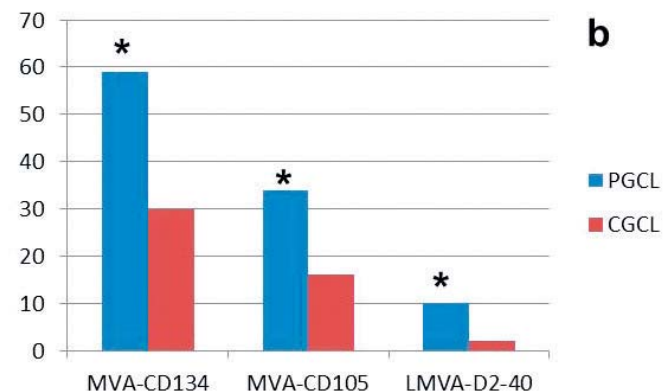

C

- PGCL

$\because \mathrm{CGCL}$

Figure 2- Comparison between PGCL and CGCL in relation to MVD and LMVD (a), MVA and LMVA (b) and percentage of FASN-positive MGC and MC (c) 


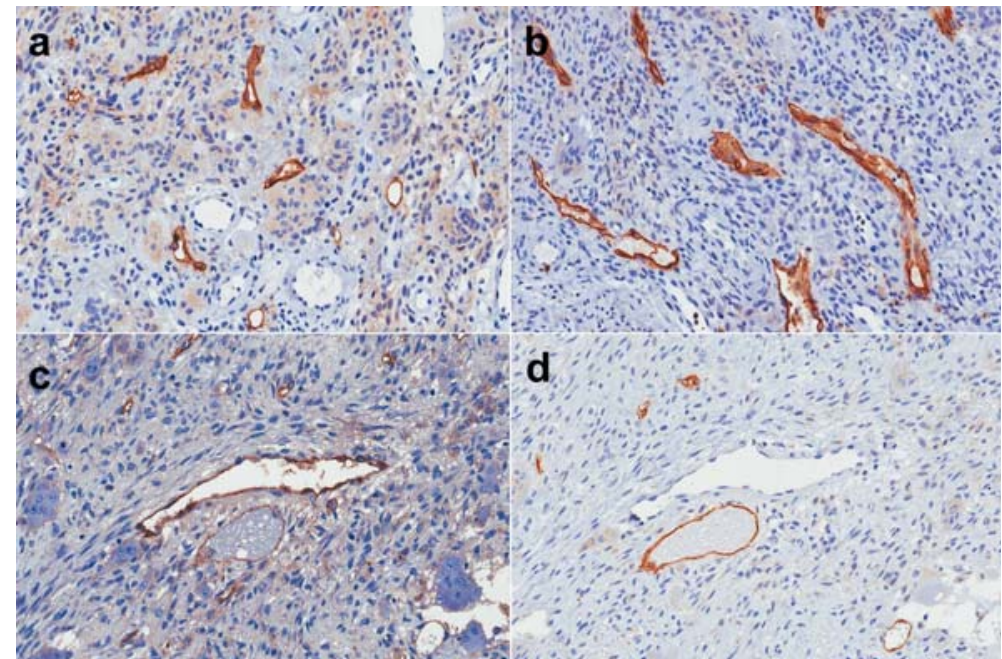

Figure 3- Immunoexpression of D2-40 in CGCL (a, 400x) and PGCL (b, 400x), notice that the lymphatic vessels are of larger diameter in PGCL than in CGCL. This case of CGCL on parallel consecutive sections presented vessels positive for both CD105 (c, 400x) and D2-40 (d, 400x), suggesting neolymphangiogenesis

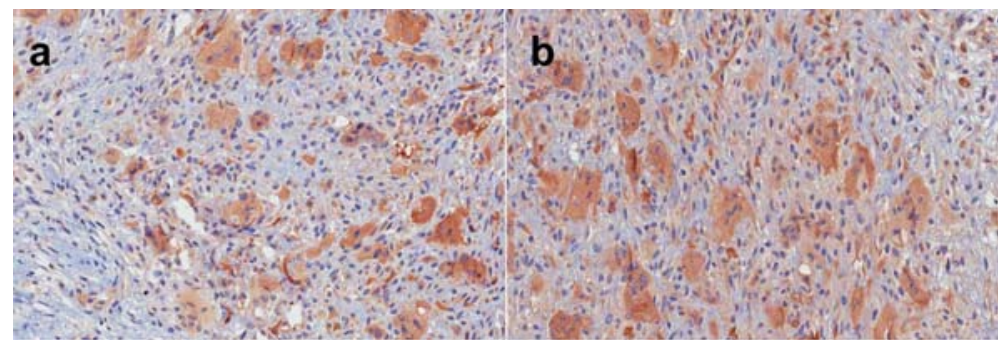

Figure 4- Immunoexpression of FASN in CGCL (a, 400x) and PGCL (b, 400x), note immunopositivity for FASN in the multinucleated giant cells and mononuclear cells in both lesions

CD105 and D2-40 in both lesions (Figures 3c, 3d), supporting the idea that some CD105-stained vessels represent newly formed lymphatic vessels.

All cases present FASN-positive multinucleated giant cells (PGCL, mean 39\%; CGCL, mean 36\%) and mononuclear cells (PGCL, mean 7\%; CGCL, mean 10\%) (Figure 2c; Figures 4a, 4b), without statistically significant differences when comparing both lesions ( $P=0.815$ and $P=0.320$, respectively). We also observed focal areas showing weak FASN immunoexpression on endothelial cells in $46 \%$ and $21 \%$ of the CGCL and PGCL cases, respectively. On the other hand, significant correlation was found between MVA-CD105 with FASN-positive mononuclear cells in CGCL $(r=0.758 ; p=0.003)$.

\section{DISCUSSION}

Both CGCL and PGCL of the jaws exhibit similar histopathological features, being characterized by numerous ovoid and spindle-shaped mononuclear cells, admixed with multinucleated giant cell, within a rich vascularized stroma ${ }^{11,16}$. Despite this, it is well known that these lesions may have different clinical behaviors ${ }^{23}$. In the current study, without significant differences between PGCL and CGCL, all cases presented FASN-positive multinucleated giant cells and mononuclear cells. Nevertheless, we observed a higher number of multinucleated giant cells than mononuclear cells (ratio 4:1) presenting FASN immunoreactivity. It is probable that cell type-specific differences in cellular metabolism may help to explain our results. Moreover, as a significant expression of vascular growth factors in multinucleated giant cells and mononuclear cells ${ }^{16}$ is most probably associated with angiogenesis in $\mathrm{GCL}$, on the other hand the lack of correlation between FASN-positive multinucleated giant cells with vascular markers observed in the current study can suggest maintenance and/or remodeling role for FASN expression in multinucleated giant cells.

Angiogenesis, which is the formation of new vessels from pre-existing vessels, is thought to be of crucial importance to the growth and maintenance of proliferative tissues ${ }^{24}$. In the oral cavity, it has been shown that the number and size of blood vessels increase from normal oral epithelium through dysplastic epithelium to reach a maximum in invasive carcinoma ${ }^{1}$, while no significant difference seems to occur between normal mucosa, hyperplasia, and dysplasia for LMVD. Conversely, invasive carcinomas presented higher LMVD 
than normal mucosa and precancerous lesions ${ }^{17}$. Regarding benign and reactive oral lesions, several studies have been performed to quantify MVD by using CD34 and CD1059,10,12,18,25-27, but none of them have assessed GCL considering both markers. In addition, although D2-40 expression has been demonstrated in normal odontogenic tissues as well as in cystic and tumor odontogenic lesions ${ }^{30}$, it is noteworthy that in GCL a stromal lymphatic vessel characterization has not been performed. Like CD105, D2-40 expression in GCL is unknown.

In the current study, we have observed uniform positivity for CD34 in all vascular structures in both lesions. Almost all our cases (93\% and 92\% of the PGCL and CGCL, respectively) exhibited CD105-positive vessels (about $32 \%$, in relation to CD34-positive vessels), while only $78 \%$ and $69 \%$ of the PGCL and CGCL, respectively, showed D2-40-positive vessels (about $10 \%$, in relation to CD34-positive vessels). Thus, the constitutive expression of CD105 in GCL supports angiogenic activity and tissue remodeling. This is further supported by FASN expression in endothelial cells, detected focally in about $46 \%$ and $21 \%$ of CGCL and PGCL cases, respectively. Moreover, the expression of endothelial markers in GCL is consistent with the presence of various angiogenic factors and matrix metalloproteinases (MMPs), such as tumor necrosis factor-alpha (TNF- $\alpha$ ), transforming growth factor-beta (TGF- $\beta$ ), basic-fibroblast growth factor (b-FGF), vascular endothelial growth factor (VEGF), and MMP-915,16,28. Since it has been shown that aggressive rather than nonaggressive subtypes of CGCL present higher MVD-CD34 ${ }^{10,18,25}$, further studies are needed in order to compare whether there are CD105 expression differences between aggressive and nonaggressive subtypes of CGCL. In relation to stromal lymphatic vessel characterization, it is interesting that lymphangiogenesis in reactive or benign oral lesions have been little studied. In the current study, we have shown that about $8 \%$ and $13 \%$ of the vessels in CGCL and PGCL group, respectively, were immunoreactive for D2-40. Although without statistically significant difference between PGCL and CGCL $(p=0.092)$, it is suggested that the higher number of lymphatic vessels detected in PGCL (Figure 2) seems to be influenced by anatomical location, since a significant number of D2-40-positive vessels could be observed at the periphery of the lesions in close association with the lamina propria. Furthermore, through consecutive section analysis, we found focal areas showing vessels stained with CD105 and D2-40, in both lesions. In order to validate our findings, we considered that the expression of CD105 and D2-40 in oral GCL should be compared with other angiogenic markers to better define neolymphangiogenesis in these lesions.
In the current study, PGCL showed a significantly greater MVA than CGCL for all vascular markers used. Similar results were shown ${ }^{27}$, which favored a reactive nature for $\mathrm{PGCL}$. It is likely that, in addition to the anatomical location of these lesions, differences in the expression pattern of angiogenic growth factors ${ }^{1,16}$ may help to explain the differences found in our study. It has been shown that FASN inhibition by orlistat may reduce endothelial cell proliferation and angiogenesis ${ }^{4}$. As previously commented, to our knowledge, the FASN immunoexpression in giant cell lesions is unknown. However, in our study, different from FASN-positive multinucleated giant cells, it is interesting to note the significant correlation found between FASN-positive mononuclear cells and MVACD105 in both lesions. Because of these findings, it is suggested that FASN-positive mononuclear cells may contribute with new vessel formation. Future studies are necessary to validate our findings and determinate possible interactions between FASN expression and angiogenic proteins in GCL.

\section{CONCLUSION}

In summary, our results suggest that greater MVD-CD34, and greater MVA-CD34, CD105 and $\mathrm{D} 2-40$, in $\mathrm{PGCL}$ rather than in $\mathrm{CGCL}$, might be associated with a reactive inflammatory process. Moreover, similar levels of FASN expression, neoangiogenesis (MVD-CD105), and lymphangiogenesis (LMVD-D2-40) between PGCL and CGCL indicate constitutive processes to regulate tissue maintenance and remodeling in both lesions.

\section{ACKNOWLEDGMENTS}

The authors would like to thank the University of Vales do Jequitinhonha e Mucuri for the master's scholarship; Victor Toral Rizo for training in applied methodology; Patrícia Correia Faria for helping with graphics; Piracicaba Dental School, Oral Diagnosis Department, for performing the immunohistochemistry; São Paulo Research Foundation (FAPESP - 2009/53839-2).

\section{CONFLICTS OF INTEREST}

The authors declare that they have no conflicts of interest in this manuscript.

\section{REFERENCES}

1- Abbas NF, Labib El-Sharkawy S, Abbas EA, Abdel Monem ElShaer M. Immunohistochemical study of p53 and angiogenesis in benign and preneoplastic oral lesions and oral squamous cell carcinoma. Oral Surg Oral Med Oral Pathol Oral Radiol Endod. 2007; 103(3):385-90. 
2- Alò PL, Visca P, Framarino ML, Botti C, Monaco S, Sebastiani V, et al. Immunohistochemical study of fatty acid synthase in ovarian neoplasms. Oncol Rep. 2000;7(6):1383-8.

3-Andrade BA, León JE, Carlos R, Delgado-Azañero W, MosquedaTaylor A, Graner E, et al. Expression of fatty acid synthase (FASN) in oral nevi and melanoma. Oral Dis. 2011;17(8):808-12. 4- Browne CD, Hindmarsh EJ, Smith JW. Inhibition of endothelial cell proliferation and angiogenesis by orlistat, a fatty acid synthase inhibitor. FASEB J. 2006;20(12):2027-35.

5- Cardoso SV, Souza KC, Faria PR, Eisenberg AL, Dias FL, Loyola AM. Assessment of angiogenesis by CD105 antigen in epithelial salivary gland neoplasms with diverse metastatic behavior. BMC Cancer. 2009;9:391.

6- Cheifetz S, Bellón T, Calés C, Vera S, Bernabeu C, Massagué J, et al. Endoglin is a component of the transforming growth factorbeta receptor system in human endothelial cells. J Biol Chem. 1992;267(27):19027-30.

7- Chirala SS, Wakil SJ. Structure and function of animal fatty acid synthase. Lipids. 2004;39(11):1045-53.

8- Chuong R, Kaban LB, Kozakewich H, Perez-Atayde A. Central giant cell lesions of the jaws: a clinicopathologic study. J Oral Maxillofac Surg. 1986;44(9):708-13.

9- Davey KJ, Perrier S, Ohe G, Gilbert AD, Bankfalvi A, Saunders $W P$, et al. Assessment of vascularity as an index of angiogenesis in periradicular granulomas: comparison with oral carcinomas and normal tissue counterparts. Int Endod J. 2008;41(11):987-96.

10- Dewsnup NC, Susarla SM, Abulikemu M, Faguin WC, Kaban LB, August M. Immunohistochemical evaluation of giant cell tumors of the jaws using CD34 density analysis. J Oral Maxillofac Surg. 2008;66(5):928-33.

11- Flórez-Moreno GA, Henao-Ruiz M, Santa-Sáenz DM, Castañeda-Peláez DA, Tobón-Arroyave SI. Cytomorphometric and immunohistochemical comparison between central and peripheral giant cell lesions of the jaws. Oral Surg Oral Med Oral Pathol Oral Radiol Endod. 2008;105(5):625-32.

12- Gadbail AR, Hande A, Chaudhary M, Nikam A, Gawande M, Patil $S$, et al. Tumor angiogenesis in keratocystic odontogenic tumor assessed by using CD-105 antigen. J Oral Pathol Med. 2011;40(3):263-9.

13- Kahn HJ, Marks A. A new monoclonal antibody, D2-40, for detection of lymphatic invasion in primary tumors. Lab Invest. 2002;82(9):1255-7.

14- Lima SC, Rizo VH, Silva-Sousa YT, Almeida LY, Almeida OP, León $\mathrm{JE}$, et al. Immunohistochemical evaluation of angiogenesis and tryptase-positive mast cell infiltration in periapical lesions. ] Endod. 2011;37(12):1642-6.

15- Matos FR, Moraes M, Nonaka CF, Souza LB, Almeida Freitas R. Immunoexpression of TNF- $\alpha$ and TGF- $\beta$ in central and peripheral giant cell lesions of the jaws. J Oral Pathol Med. 2012;41(2):194-9. 16- Matos FR, Nonaka CF, Miguel MC, Galvão HC, Souza LB, Freitas RA. Immunoexpression of MMP-9, VEGF, and VWF in central and peripheral giant cell lesions of the jaws. J Oral Pathol Med. $2011 ; 40(4): 338-44$.
17- Palomba A, Gallo O, Brahimi A, Franchi A. Evaluation of lymphangiogenesis in premalignant conditions of the head and neck mucosa. Head Neck 2010;32(12):1681-5.

18- Peacock ZS, Jordan RC, Schimidt BL. Giant cell lesion of the jaws: does the level of vascularity and angiogenesis correlate with behavior? J Oral Maxillofac Surg. 2012;70(8):1860-6.

19- Pizer ES, Jackisch C, Wood FD, Pasternack GR, Davidson NE, Kuhajda FP. Inhibition of fatty acid synthesis induces programmed cell death in human breast cancer cells. Cancer Res. 1996;56(12):2745-7.

20- Rossi S, Graner E, Febbo P, Weinstein L, Bhattacharya N, Onody $\mathrm{T}$, et al. Fatty acid synthase expression defines distinct molecular signatures in prostate cancer. Mol Cancer Res. 2003;1(1):707-15. 21- Seguin F, Carvalho MA, Bastos DC, Agostini M, Zecchin KG, Alvarez-Flores MP, et al. The fatty acid synthase inhibitor orlistat reduces experimental metastases and angiogenesis in B16-F10 melanomas. $\mathrm{Br}$ J Cancer. 2012;107(6):977-87.

22- Silva SD, Cunha IW, Nishimoto IN, Soares FA, Carraro DM, Kowalski LP, et al. Clinicopathological significance of ubiquitinspecific protease 2a (USP2a), fatty acid synthase (FASN), and ErbB2 expression in oral squamous cell carcinomas. Oral Oncol. 2009;45(10):e134-9.

23- Souza PE, Mesquita RA, Gomez RS. Evaluation of p53, PCNA, $\mathrm{Ki}-67, \mathrm{MDM} 2$ and AgNOR in oral peripheral and central giant cell lesions. Oral Dis. 2000;6(1):35-9.

24- Spiegelaere W, Casteleyn C, Van den Broeck W, Plendl J, Bahramsoltani $M$, Simoens $P$, et al. Intussusceptive angiogenesis: a biologically relevant form of angiogenesis. J Vasc Res. 2012;49(5):390-404.

25- Susarla SM, August M, Dewsnup N, Faquin WC, Kaban LB, Dodson TB. CD34 staining density predicts giant cell tumor clinical behavior. J Oral Maxillofac Surg. 2009;67(5):951-6.

26- Vasconcelos MG, Alves PM, Vasconcelos RG, Silveira EJ, Medeiros AM, Queiroz LM. Expression of CD34 and CD105 as markers for angiogenesis in oral vascular malformations and pyogenic granulomas. Eur Arch Otorhinolaryngol. 2011;268(8):1213-7.

27- Vassilopoulos SI, Tosios KI, Panis VG, Vrostsos JA. Endothelial cells of oral pyogenic granulomas express eNOS and CD105/ endoglin: an immunohistochemical study. J Oral Pathol Med. 2011;40(4):345-51.

28- Vered M, Buchner A, Dayan D. Giant cell granuloma of the jawbones: a proliferative vascular lesion? Immunohistochemical study with vascular endothelial growth factor and basic fibroblastic growth factor. J Oral Pathol Med. 2006;35(10):613-9.

29- Weiss L, Hoffmann GE, Schreiber R, Andres H, Fuchs E, Körber $E$, et al. Fatty-acid biosynthesis in man, a pathway of minor importance. Purification, optimal assay conditions, and organ distribution of fatty-acid synthase. Biol Chem Hoppe Seyler. 1986;367(9):905-12.

30- Zustin J, Scheuer HA, Friedrich RE. Podoplanin expression in human tooth germ tissues and cystic odontogenic lesions: an immunohistochemical study. J Oral Pathol Med. 2010;39(1):11520. 